\title{
Los comités de investigación y ética en investigación y la obligación de que operen de acuerdo con el principio de la alianza social
}

\section{Edith Valdez-Martínez ${ }^{*}$ y Miguel Bedolla²}

${ }^{1}$ Instituto Mexicano del Seguro Social, Coordinación de Investigación en Salud, Ciudad de México, México; ${ }^{2}$ Northwest Vista College, San Antonio, Texas, Estados Unidos

\section{Resumen}

La relación entre alianza social, ética e investigación científica es extremadamente importante para la sociedad. La prosperidad económica y la mejor salud son dos de las principales razones por las cuales la sociedad apoya a la ciencia, sin que la sociedad misma pueda determinar la naturaleza de las investigaciones que serán implementadas; esto último lo deciden los comités de investigación (Cl) y los comités de ética en investigación (CEI). En este artículo se analiza cómo el trabajo de los $\mathrm{Cl}$ y CEI debe tener una alianza social y representar los intereses de la sociedad para promover la confianza de esta en la investigación.

PALABRAS CLAVE: Comités de investigación. Comités de ética. Alianza social. Ética. México.

\section{Research and research ethics committees and the obligation for them to operate in accordance with the principle of the social covenant}

\section{Abstract}

The relationship between the social covenant, ethics and scientific research is highly important for society. Economic prosperity and better health are two of the main reasons why society supports science, without society itself being able to determine the nature of the research that is to be implemented; this is decided by Research Committees (RCs) and Research Ethics Committees (RECS). This article analyzes how the work of RCs and RECs must have a social covenant and represent the interests of society in order to promote its trust in research.

KEY WORDS: Research committees. Ethics committees. Social covenant. Ethics. Mexico.

\section{Introducción}

La existencia implícita de la alianza social es precisamente la razón por la cual los antiguos griegos llamaban a la ética "la ciencia política" (episteme politike), es decir, la ciencia de la ciudad o la ciencia para ser buen ciudadano. ${ }^{1}$ En la sociedad del mundo moderno, la ciencia, como un proceso sistemático de racionalidad, incrementa su importancia cuando involucra a la investigación, porque de ella resultan beneficios prácticos para la sociedad en general. Por su parte, la sociedad, a través de decisiones de política pública, designa recursos económicos a la investigación para acelerar el progreso científico, sin que pueda determinar la naturaleza de las investigaciones que serán implementadas; esto último lo deciden los
Correspondencia:

*Edith Valdez-Martínez

E-mail: edith.valdez@imss.gob.mx
Fecha de recepción: 05-09-2019

Fecha de aceptación: 30-09-2019

DOI: 10.24875/GMM.19005512
Gac Med Mex. 2020;156:139-142

Disponible en PubMed

www.gacetamedicademexico.com

0016-3813/@ 2019 Academia Nacional de Medicina de México, A.C. Publicado por Permanyer. Este es un artículo open access bajo la licencia CC BY-NC-ND (http://creativecommons.org/licenses/by-nc-nd/4.0/). 
comités de investigación $(\mathrm{Cl})$ junto con los de ética en investigación (CEI). En la medida en la que el trabajo de los $\mathrm{Cl}$ y CEI sea una actividad realizada por humanos, las decisiones de los comités reflejarán las emociones, los valores y las creencias de sus miembros, lo cual incide en la manera como la ciencia es percibida e implementada y como las investigaciones son leales a la alianza social.

\section{La alianza social, un principio inherente a la ciencia}

La palabra alianza deriva de alligare, palabra latina que indica atar. El diccionario de la Real Academia Española la define como "la acción de aliarse". ${ }^{2}$ Los investigadores y los miembros de los $\mathrm{Cl}$ y $\mathrm{CEI}$ deben tener a la lealtad como la obligación que da significado a la alianza social. Ellos tienen, por lo tanto, responsabilidades fiduciarias. El cumplimiento responsable de los compromisos fundamentales (de donde emana la lealtad) de los investigadores y de los $\mathrm{Cl}$ y $\mathrm{CEI}$ fortalece la confianza de la sociedad en la investigación científica. Cuando el propio interés de alguna de las partes amenaza o viola los legítimos beneficios de la otra, se vulnera el objetivo primario de la investigación científica: generar información válida, con o sin ramificaciones prácticas inmediatas, que beneficie a la sociedad. ${ }^{3,4}$

Actualmente por ley, en México únicamente las propuestas de investigación aprobadas por los $\mathrm{Cl}$ y CEI pueden ser implementadas. La toma de decisiones de los miembros de los $\mathrm{Cl}$ y CEI en la evaluación de proyectos de investigación comprende más que las obligaciones teóricas expresadas en la ley, en las normas y en los principios éticos; involucra también los rasgos del carácter de los miembros de los $\mathrm{Cl}$ y CEl. ${ }^{5}$ De ahí que no sorprenda que el trabajo de los $\mathrm{Cl}$ y $\mathrm{CEI}$ pueda resultar en ocasiones controversial, especialmente cuando rechazan protocolos de investigación con ideas innovadoras y con la posibilidad de generar resultados que mejorarían el sistema de salud del país, porque los miembros de los $\mathrm{Cl}$ y CEI no entienden los métodos con los que serán llevadas a cabo las investigaciones. Esta ruptura entre la alianza social y los $\mathrm{Cl}$ y CEI también ocurre cuando estos dejan de funcionar como un organismo crítico y con clara responsabilidad social y sesgan sus juicios por deferencia hacia un investigador o grupo de investigadores que son percibidos con autoridad 0 reputación.

Cuando los $\mathrm{Cl}$ y CEl soslayan su responsabilidad de alianza social durante la evaluación de los protocolos de investigación, abandonan la probabilidad de promover mejoras en la salud, en el bienestar o en el conocimiento de la población. El siguiente es un ejemplo real y actual en el que se soslaya la alianza social:

- Protocolo de investigación multicéntrico de diseño transversal descriptivo, exploratorio, retrospectivo, que platea una pregunta de investigación original y relevante para la mejora de la calidad de vida de mujeres adolescentes con discapacidad intelectual. Como parte de las técnicas de recolección de información, en la metodología se menciona la revisión de expedientes clínicos con el único propósito de generar información referente a la higiene menstrual de este grupo de mujeres. En el protocolo se especifica adecuadamente cómo se mantendrá el anonimato y la confidencialidad de los datos personales. En su dictamen escrito, el $\mathrm{Cl}$ y el CEl del hospital consideraron que la propuesta de investigación era científicamente relevante, que estaba metodológicamente bien diseñada y que cumplía con los requisitos éticos; no obstante, rechazaron el protocolo argumentando que en el hospital nunca se habían realizado histerectomías en mujeres con discapacidad intelectual.

Ante la aserción del $\mathrm{Cl}$ y $\mathrm{CEI}$ de que ninguna histerectomía fue encontrada en mujeres con discapacidad intelectual cabe preguntarse ¿cómo garantizaron el rigor científico y validez de los resultados de su búsqueda?, ¿está justificado que los miembros del $\mathrm{Cl}$ y CEI hayan implementado motu proprio la investigación sin previa autorización de los autores del protocolo?, ¿cómo mantuvieron el rigor científico y ético sin haber seguido el protocolo que ellos mismos no aprobaron?

Al ofrecer sus servicios, los $\mathrm{Cl}$ y CEl están asumiendo la responsabilidad fiduciaria y la responsabilidad de actuar de acuerdo con criterios establecidos nacional e internacionalmente. Su actuar se vuelve idiosincrático cuando no procede en consonancia con estas responsabilidades, lo que, por consiguiente, impide que se cumplan los beneficios que para la sociedad representa la alianza social.

\section{La autoría y su relación con la alianza social}

Se considera autor a quien ha realizado contribuciones intelectuales sustantivas al protocolo de una investigación y luego al artículo en el que se publicarán los resultados. Quien aparece como autor 
debe asumir la responsabilidad de las siguientes tres condiciones: ${ }^{6}$

1. Hacer aportaciones importantes al diseño o recolección de datos o análisis.

2. Realizar revisión crítica y sustancial del contenido intelectual.

3. Aprobar la obra terminada.

Los nombres de los colaboradores que no cumplen con los tres criterios de autoría deben aparecer en la sección de agradecimientos. ${ }^{6}$

La autoría conlleva importantes implicaciones y, por ende, presiones académicas, sociales y económicas que avivan ansiedades profundamente establecidas en los individuos y que al interactuar con el carácter de cada uno definen un área de posibles comportamientos. En las unidades hospitalarias, los directores o jefes de investigación y los investigadores están obligados simultáneamente a maximizar la productividad científica. Esta poderosa presión es lo que, en muchos casos, define a la moral (del latín moralis, lo que se acostumbra²) de la práctica de la investigación científica. Un caso real que ilustra una práctica común y generalizada:

- Una investigación sectorial multicéntrica debe contar con la aprobación de los $\mathrm{Cl}$ y CEl de cada centro participante. Un prerrequisito que exigen los directores o jefes de investigación (para que el proyecto pueda ser enviado a evaluación por los $\mathrm{Cl}$ y CEI de su respectivo hospital) es que en el protocolo se nombre a un médico o profesional de la salud adscrito al potencial hospital participante como "investigador responsable", con la instrucción de que ese "investigador responsable" deberá aparecer como autor. Este mismo requisito está establecido en los formatos (de cada hospital invitado) que el investigador debe rellenar para someter el protocolo a evaluación por los comités. Si bien esto es la moral imperante, es decir, lo que se acostumbra, la infracción ética ocurre por el hecho de que esos "investigadores responsables" de los hospitales participantes, que aparecerán en la lista de autores, en ocasiones únicamente favorecen o permiten el acceso a la información o a las bases de datos o a los expedientes clínicos, actividades que no justifican la condición de autor en una investigación. No obstante, el incumplimiento de este prerrequisito hace que un protocolo sea de inicio rechazado bajo el argumento de que "sus hospitales no son únicamente proveedores de datos 0 muestras".
La autoría injustificada da lugar a una serie de deliberaciones éticas: por un lado, siempre es una mentira y, por consiguiente, una infracción ética, pues un individuo obtiene crédito por algo que no ha hecho y que puede utilizar para conseguir un beneficio personal. Por otra parte, es testimonio del carácter 0 forma de pensar y ser de los agentes involucrados, a la luz de las expectaciones, valores e intereses que los motivan, y de la manera como lo anterior influye en sus juicios y decisiones. ${ }^{5}$ Asimismo, representa un ansia por aumentar la productividad (publicar a toda costa), lo cual socava el cimiento científico en el que debe basarse la investigación: la búsqueda responsable de la verdad. Al rechazar un protocolo cuyos autores legítimos se niegan a obedecer el mandato de asignar una autoría espuria, los Cl y CEI dejan de promover la generación de conocimiento científico al cual están obligados por la alianza social.

\section{La lealtad profesional de los $\mathrm{Cl}$ y CEI}

En México, los grupos que independientemente evalúan los protocolos de investigación son tres: $\mathrm{Cl}, \mathrm{CEI}$ y comités de bioseguridad. ${ }^{7,8}$ Hemos enfocado este artículo en los dos primeros por ser los que generalmente evalúan el mayor número de protocolos y por tener funciones superpuestas. Brevemente: para que un $\mathrm{CEl}$ determine que una propuesta de investigación es ética, debe evaluar la importancia social y científica de esta, la validez y plausibilidad metodológica, la conveniencia de los criterios de selección, la razón riesgo/beneficio y los documentos del consentimiento informado, ${ }^{4,9}$ criterios que también deben ser examinados por el $\mathrm{Cl}^{7}{ }^{7}$ No está definida la razón por la cual deben existir dos grupos con actividades superpuestas. La esencia del trabajo de los CEI no debe reducirse al consentimiento informado de los sujetos de investigación. ${ }^{4,9}$ Tampoco los aspectos metodológicos evaluados por los $\mathrm{Cl}$ son ajenos a la ética. ${ }^{4} \mathrm{La}$ ética de la investigación está inmersa en la investigación misma, de ahí que tanto los $\mathrm{Cl}$ como los $\mathrm{CEl}$ estén para mantener altos estándares éticos, definidos nacional e internacionalmente, en la conducción de una investigación a fin de lograr su propósito: la alianza social.

Antes de ser implementados, los estudios multicéntricos deben ser aprobados por los $\mathrm{Cl}$ y $\mathrm{CEI}$ de cada centro participante. La variedad de los argumentos para rechazar o aprobar con modificaciones una investigación indica como los $\mathrm{Cl}$ y $\mathrm{CEI}$ de los diferentes centros ven una misma propuesta. Las diferencias 
en las justificaciones dependen del carácter o forma de ser de los $\mathrm{Cl}$ y CEI. Para ilustrar lo anterior regresemos al primer caso, por ser una propuesta de investigación multicéntrica:

- En un hospital, los Cl y CEl rechazaron el protocolo debido a "nunca haber realizado histerectomías en mujeres con discapacidad intelectual"; en otro, lo objetaron por "la posible existencia de situaciones riesgosas para el hospital". Ambos hospitales son centros de referencia en atención médica e investigación; otros hospitales aprobaron la propuesta. Al denegar la propuesta de investigación, los $\mathrm{Cl}$ y $\mathrm{CEI}$ vulneraron su lealtad profesional (inmersa en la alianza social) al conferirle primacía a los intereses de los hospitales a los que pertenecen, en lugar de considerar los de la población de mujeres que sería beneficiada con los resultados de la investigación. El mejor interés de los enfermos puede no ser el del hospital, el cual pudiera estar más interesado en evitar aspectos legales, juicios u opiniones de la misma comunidad científica o médica.

En suma, los $\mathrm{Cl}$ y $\mathrm{CEI}$ contribuirán poco a la mejora de la salud y el bienestar individual y colectivo de los servicios de salud si no comprenden la naturaleza de su trabajo y su relación con la alianza social. La lealtad profesional emerge del carácter (ethos) de cada miembro de los $\mathrm{Cl}$ y $\mathrm{CEI}$ y de los compromisos fundamentales que individualmente y como un todo tienen con la sociedad.

\section{Conclusión}

Aunque existen guías nacionales para la integración y funcionamiento de los $\mathrm{CEI}$ y $\mathrm{Cl}$, la realidad actual del trabajo de los comités resalta la necesidad de cambios, para así favorecer que funcionen en congruencia con la alianza social y la ética de la investigación científica.

\section{Bibliografía}

1. Voegelin E. Plato and Aristotle. En: Voegelin E, editor. Order and History. Vol. 3. EE. UU.: Louisiana State University Press; 1957.

2. Diccionario de la lengua española: España: Real Academia Española; 2001.

3. Declaración de Helsinki. EE. UU.: Asociación Médica Mundial; 2013.

4. Emanuel EJ, Wendler D, Grady C. What makes clinical research ethical? JAMA. 2000;283:2701-2711.

5. Valdez-Martínez E, Bedolla M. Los comités de investigación en salud: ¿de dónde surge su autoridad, su responsabilidad fundamental, y la necesidad de que se les haga una auditoría periódica? Gac Med Mex. 2019.

6. International Committee of Medical Journal Editors [sitio web]. Defining the role of authors and contributors. [Actualizado 2020]. Disponible en: http://www.icmje.org/recommendations/browse/roles-and-responsibilities/defining-the-role-of-authors-and-contributors.html

7. Norma oficial mexicana NOM-012-SSA3-2012, que establece los criterios para la ejecución de proyectos de investigación en salud en seres humanos. México: Diario Oficial de la Federación; 2013 Ene 4.

8. Decreto por el que se adiciona el artículo 41 bis y se reforma el artículo 98 de la Ley General de Salud. México: Diario Oficial de la Federación; 2011 Dic 14.

9. Guía nacional para la integración y el funcionamiento de los comités de ética en investigación. México: Comisión Nacional de Bioética/Secretaria de Salud; 2018. 